\title{
El aprendizaje basado en problemas en estudiantes universitarios
}

\author{
Problem-based learning in university students
}

\author{
María Cruz Lozano-Ramírez \\ e-mail: ma.cruz.lozano.ramirez@uabc.edu.mx \\ Universidad Autónoma de Baja California. México
}

\begin{abstract}
Resumen
Este artículo presenta los resultados de un estudio sobre el Aprendizaje basado en Problemas (ABP) como estrategia didáctica en estudiantes de pregrado. El objetivo de esta investigación fue identificar las experiencias de los estudiantes en la solución de un problema real y explicar su contribución en la formación profesional. El diseño de investigación fue no experimental y el estudio explicativo, aplicado a una muestra de 124 estudiantes en una Institución de Educación Superior (IES) ubicada en la Ciudad de Tijuana, B. C., México. Los resultados de las pruebas señalan que el Aprendizaje Basado en Problemas les demanda a los estudiantes capacidades de análisis y reflexión para abordar un problema de manera objetiva, crítica y creativa. Así como la toma de decisiones para determinar expectativas y definir metas para la solución de este. Los resultados también explican que esta estrategia les provee de conocimientos para la evaluación de problemas cotidianos, familiares y académicos y les aporta habilidades de negociación, de comunicación y experiencias que fortalecen su formación profesional. Dichas experiencias contribuyen en la comprensión de materias y les permiten desarrollar estrategias de estudio. Si bien el Aprendizaje Basado en Problemas es altamente beneficioso para los estudiantes, el estudio concluye que como técnica es poco empleada por los profesores por lo que se recomienda a las IES efectuar un inventario de las estrategias didácticas implementadas por los profesores y desarrollar procesos de capacitación para complementar y respaldar su práctica docente.
\end{abstract}

Palabras clave: aprendizaje basado en problemas; estrategias de aprendizaje; procesos de aprendizaje; educación universitaria.

\begin{abstract}
This article presents the results of a study on Problem-Based Learning (ABP in Spanish) as a teaching strategy in undergraduate students. The objective of this research was to identify the experiences of students in solving a real problem and explain their contribution in professional training. The research design was non-experimental, and the explanatory study was applied to a sample of 124 students at an Institution of Higher Education (IES in Spanish) located in the City of Tijuana, B.C., Mex. The results of the tests indicate that Problem-Based Learning demands students' analysis and reflection skills to approach a problem objectively, critically and creatively. As well as decision making to determine expectations and define goals for its solution. The results also explain that this strategy provides them with knowledge for the evaluation of daily, family and academic problems and provides them with negotiation, communication and experience skills that strengthen their professional training. These experiences contribute to the understanding of subjects and allow them to develop study strategies. Although Problem-Based Learning is highly beneficial for students, the study concludes that as a technique it is little used by teachers, so it is recommended to the IESs to make an inventory of the teaching strategies implemented by teachers and develop training processes to complement and support their teaching practice.
\end{abstract}

Keywords: learning; problem-based learning; learning strategies; learning processes; university education.

Recibido / Received: 15-01-2020

Aceptado / Accepted: 07-05-2020

Publicado en linea / Published online: 22-12-2020

Cómo referenciar este artículo / How to reference this article:

Lozano-Ramírez, M. C. (2021). El aprendizaje basado en problemas en estudiantes de pregrado. Tendencias Pedagógica 37, pp. 90-103. doi: 10.15366/tp2021.37.008 


\section{Introducción}

El Aprendizaje Basado en Problemas es una estrategia didáctica centrada en el aprendizaje, la investigación y la reflexión crítica para encontrar soluciones a un problema propuesto (Fernández, Fernández \& Polo, 2017; Gómez, 2005 cit. en Rodríguez, Ordoñez, \& Meneses, 2018). Su principal objetivo es la adquisición de nuevos conocimientos (Walker, Bridges, \& Chan, 1996) para abordar un problema y soluciones para cada una de sus fases (Hemker, Prescher, \& Narciss, 2017). Para ello, es aplicado bajo principios basados en el rol que desempeñan los estudiantes (Galindo, Arango, Díaz, Villegas, Aguirre, Kambourova, \& Jaramillo, 2011; Giraldo, Giraldo, \& Valderrama, 2018), a quienes se les presenta un caso (problema) para que a través de una discusión en equipo compartan conocimientos para la solución de este (Costas \& Nicolaou, 2018). Como parte del currículo, el Aprendizaje Basado en Problemas se identifica en materias cuyos contenidos demandan la solución de problemas lógicos, matemáticos, algorítmicos, estratégicos o de toma de decisiones (Gorbaneff, 2010; Gros, 1990; Ortega \& Carrascal, 2017), donde la reflexión, el autocontrol y la voluntad requieren habilidades para solucionar problemas reales (Etherington, 2011). Por ende, la construcción del aprendizaje considera al alumno como responsable de su aprendizaje ya que «El alumno es quién identifica objetivos, se compromete, descubre, desea conocer más y así se retroalimenta el proceso y se estimula la capacidad de liderazgo, de comunicación y toma de decisiones, la creatividad, el pensamiento crítico y el trabajo en equipo» (González, Carbonero, Lara, \& Martín, 2014).

Por ello, en su aplicación debe considerarse el contexto profesional y personal del estudiante (Relevancia), la motivación hacia la curiosidad intelectual para documentar y efectuar el análisis de la información para la solución del problema (Cobertura) a partir de diversas alternativas (Complejidad). Lo anterior implica que los profesores y tutores que efectúan el acompañamiento deben ser especialistas en los métodos del programa, dominio de la interacción de grupos, coordinación y autoevaluación, flexibilidad, disposición para atender las necesidades de los estudiantes y conocimiento del método científico (Restrepo Gómez, 2005; 2015 cit., en Gil, 2018). Esto se retoma desde la perspectiva del currículo, en función de los atributos que deben tener los profesores ya que a partir de ello efectúan el rediseño a nivel institucional. En uno u otro sentido, su concepción como método o estrategia dependerá del impacto curricular y aplicación práctica (Travieso \& Ortiz, 2018). Por lo anterior, este estudio define como objetivo de investigación identificar las experiencias, conocimientos y habilidades adquiridas por los estudiantes de pregrado con la estrategia didáctica Aprendizaje basado en problemas para explicar su contribución en la formación profesional. El estudio es importante y servirá a las Instituciones de Educación Superior (IES) porque la experiencia adquirida por los estudiantes suministrará elementos para el diseño y reestructuración de contenidos con aprendizajes significativos. Como relevancia social, el estudio beneficiará a las IES porque podrán desarrollar programas de entrenamiento dirigidos al profesorado sobre el diseño de problemas para su aplicación en el aula. A su vez, los profesores contarán con información del impacto de la estrategia en la adquisición de conocimientos, habilidades, competencias y podrán robustecer el acompañamiento y monitorear su desempeño porque en el medio plazo y través de la solución de problemas esto favorecerá su inserción en el ámbito laboral. Como valor teórico, el estudio explicará las diversas aportaciones teóricas y posibles relaciones de las variables Aprendizaje basado en Problemas y Adquisición de conocimientos y habilidades. Como utilidad metodológica, el estudio proveerá un instrumento de medición (Cuestionario) para su réplica en otros contextos geográficos.

\section{Perspectiva teórica del Aprendizaje Basado en Problemas}

La implementación del Aprendizaje Basado en Problemas suele plantear algunos desafíos en la interacción de los estudiantes ya que se desarrolla bajo entornos de reflexión y cuestionamiento permanente para incentivar la tolerancia a las críticas, la responsabilidad y la disciplina personal en favor de la mejor solución a un problema (Olmedo et al., 2016). Esto les permite asimilar conocimientos y habilidades para desarrollar capacidades que apoyen la construcción de su aprendizaje (Geitz, Brinke, \& Kirschner, 2016; Barbosa, Kruta, Rodríguez, \& Felipe, 2018) por lo que bajo un enfoque integral del problema (Sainz \& Fernández, 2012) se impulsa la competitividad, a la vez que se convierte en un pilar fundamental (Lima et al., 2017).

Como estrategia didáctica, el Aprendizaje Basado en Problemas provee técnicas y procedimientos para orientar el aprendizaje hacia el logro de los objetivos de enseñanza (Carrasco, 2004) a través de 
la colaboración (Lee et al., 2017) y otorga certidumbre a los procesos porque se conocen los estilos de aprendizaje y es posible aplicar diagnósticos (Teruel, Domínguez, \& Fernández, 2015) que mejoren el diseño de estrategias presenciales, instruccionales, de aprendizaje o evaluación (Feo, 2010). De acuerdo con Kolb (Kolb cit. en Rodríguez et al., 2018), los individuos desarrollan estrategias considerando sus propios intereses, experiencias, vivencias y oportunidades ya que se basan en su percepción por lo que se enfocan en el aprendizaje y reflexionan las decisiones sobre la mejor manera de abordar un problema.

En ello, el Aprendizaje Basado en Problemas incorpora la teoría y la práctica favoreciendo los procesos de aprendizaje de los estudiantes (Carrió et al., 2018) porque desarrollan conocimientos y adquieren habilidades para el planteamiento de problemas, objetivos e hipótesis (Hemker et al., 2017; Sepúlveda, Cabezas, García, \& Fonseca, 2019), así como habilidades interpersonales en la comunicación oral y escrita (ABP, s/f; Fernández, López, Fernández, \& Polo, 2014). Aunado a lo anterior, esta estrategia respalda el pensamiento crítico y la interacción con otras disciplinas (Makin, 2015) porque los estudiantes deciden qué aprender con el apoyo del profesor, tutor o facilitador, quienes los guían en los procesos de aprendizaje del equipo que desconoce las causas que lo originan y provee la estructura para su solución (Gregori\&y Menéndez, 2015; Lee et al., 2017; Costas \& Nicolaou, 2018; Travieso \& Ortiz, 2018).

Aunque el Aprendizaje Basado en Problemas aporta a los estudiantes amplias ventajas en la adquisición de conocimientos, habilidades y técnicas, también es necesario destacar que su implementación no está exenta de obstáculos donde profesores y estudiantes coexisten en una cultura educativa basada en planes de estudio y métodos que privilegian la transmisión de conocimientos sobre la adquisición de competencias. Por ejemplo, un estudio (Carrió et al., 2018) mostró que los estudiantes consideran que es útil pero propicia un aprendizaje fragmentado que les absorbe más tiempo, motivo por el cual prefieren las clases expositivas. Los estudiantes también comentaron que el diseño del problema les dificulta identificar los objetivos de aprendizaje por lo que los problemas breves les resultan más atractivos. Asimismo, consideran que los facilitadores/tutores deben unificar criterios de evaluación y propiciar un mayor acompañamiento. Aplicado a la enseñanza de idiomas, el impacto en el pensamiento crítico es limitado (Ding, 2016). En el caso de Ucrania, uno de los principales problemas con la implementación son los objetivos y entornos de aprendizaje ya que la rigidez de la estructura universitaria, la flexibilidad y multidisciplinariedad del Aprendizaje Basado en Problema es incompatible con los enfoques empleados en la educación formal (Dmitrenko, 2016). No obstante, se debe considerar que para este tipo de estrategias de aprendizaje los estudiantes deben investigar y combinar la teoría con la búsqueda de soluciones ya que el trabajo colegiado contribuye a la adquisición de conocimientos, genera actitudes positivas y mejora sus habilidades (Edwards, Kupczynski, \& Groff, 2019).

\section{Método}

\subsection{Diseño de investigación}

El diseño de investigación fue no experimental y el estudio explicativo de corte cuantitativo, aplicado a una muestra de conveniencia de 124 estudiantes de $4 .^{\circ}$ semestre que cursaron la materia de Metodología de la Investigación de la Carrera de Mercadotecnia en una Institución de Educación Superior ubicada en la Ciudad de Tijuana, Baja California, Méx. La hipótesis de investigación se definió como: «Hi: El aprendizaje basado en problemas proporciona experiencias, conocimientos y habilidades para la solución de problemas reales y fortalece la formación profesional en estudiantes de pregrado».

Para su medición se operacionalizó la variable Aprendizaje basado en Problemas a través de un cuestionario de 16 ítems, obteniendo una consistencia interna de 0.880 de confiabilidad (tabla 1).

Tabla 1.

Operacionalización de la variable «aprendizaje basado en problemas»

\begin{tabular}{|c|c|c|c|c|}
\hline Definición conceptual & Definición operacional & Ítems & $\begin{array}{l}\text { Total de } \\
\text { ítems }\end{array}$ & $\begin{array}{l}\text { Alpha de } \\
\text { Cronbach }\end{array}$ \\
\hline
\end{tabular}




\begin{tabular}{|c|c|c|c|}
\hline $\begin{array}{l}\text { Estrategia de enseñanza } \\
\text { que promueve la adqui- } \\
\text { sición de conocimien- } \\
\text { tos, el desarrollo de ha- } \\
\text { bilidades y actitudes } \\
\text { para el análisis y solu- } \\
\text { ción de problemas }\end{array}$ & $\begin{array}{l}\text { Aplicación de un cuestiona- } \\
\text { rio tipo escala Likert de } 7 \\
\text { puntos que aborda la per- } \\
\text { cepción de los sujetos sobre } \\
\text { su experiencia en la solu- } \\
\text { ción del problema Informa- } \\
\text { ción Verde }\end{array}$ & $\begin{array}{l}\text { P9, P10, P11, } \\
\text { P12, P13, P15, } \\
\text { P16, P17, P18, } \\
\text { P19, P20, P21, } \\
\text { P22, P25, P26, } \\
\text { P27 }\end{array}$ & $(0.880)$ \\
\hline
\end{tabular}

Fuente: elaboración propia.

A su vez, la variable Adquisición de conocimientos y habilidades se compone de 20 ítems de los cuales se reporta una consistencia interna de 0.909 (tabla 2).

Tabla 2.

Operacionalización de la variable «adquisición de conocimientos y habilidades»

\begin{tabular}{|c|c|c|c|c|}
\hline Definición conceptual & Definición operacional & Items & $\begin{array}{l}\text { Total de } \\
\text { ítems }\end{array}$ & $\begin{array}{l}\text { Alpha de } \\
\text { Cronbach }\end{array}$ \\
\hline $\begin{array}{l}\text { Estructura de conoci- } \\
\text { mientos que funda- } \\
\text { menta la formación } \\
\text { profesional de alum- } \\
\text { nos de pregrado }\end{array}$ & $\begin{array}{l}\text { Aplicación de un cuestionario } \\
\text { tipo escala Likert de } 7 \text { puntos } \\
\text { que aborda la percepción de } \\
\text { los sujetos respecto al desa- } \\
\text { rrollo y aplicación de conoci- } \\
\text { mientos, técnicas y habilida- } \\
\text { des para la definición, evalua- } \\
\text { ción, recopilación y análisis de } \\
\text { datos para resolver un pro- } \\
\text { blema }\end{array}$ & $\begin{array}{l}\text { P14, P23, P24, } \\
\text { P28, P30, P31, } \\
\text { P32, P33, P34, } \\
\text { P35, P36, P37, } \\
\text { P38, P39, P40, } \\
\text { P41, P42, P43, } \\
\text { P44, P45 }\end{array}$ & 20 & $(0.909)$ \\
\hline
\end{tabular}

Fuente: elaboración propia.

Las variables demográficas fueron evaluadas con un cuestionario de 8 preguntas que abordan: edad, género, estado civil, escolaridad y actividad laboral (tabla 3).

Tabla 3.

Operacionalización de variables demográficas

\begin{tabular}{|c|c|c|c|}
\hline Variable & Indicador & Tipo de variable & $\begin{array}{l}\text { Nivel de } \\
\text { medición }\end{array}$ \\
\hline Edad & $\begin{array}{l}\text { Años cumplidos al momento de con- } \\
\text { testar el instrumento de medición. }\end{array}$ & Cuantitativa discontinua & Ordinal \\
\hline Género & $\begin{array}{l}\text { - Masculino } \\
\text { - Femenino }\end{array}$ & Cualitativa & Nominal \\
\hline Escolaridad & Formación profesional en proceso & Cualitativa ordinal & Nominal \\
\hline Estado civil & $\begin{array}{l}\text { - Casado } \\
\text { - Soltero } \\
\text { - } \text { Divorciado } \\
\text { - Unión Libre }\end{array}$ & Cualitativa & Nominal \\
\hline Actividad laboral & $\begin{array}{l}\text { - Estatus laboral de los sujetos } \\
\text { - Con actividad laboral } \\
\text { - Sin actividad laboral }\end{array}$ & Cualitativa & Nominal \\
\hline Edad & $\begin{array}{l}\text { Años cumplidos al momento de con- } \\
\text { testar el instrumento de medición }\end{array}$ & Cuantitativa discontinua & Ordinal \\
\hline
\end{tabular}

Fuente: elaboración propia.

\subsection{Aplicación de la estrategia didáctica Aprendizaje Basado en Problemas}

La integración de equipos colaborativos. Para iniciar la actividad y propiciar la reflexión e intercambio de ideas (Campoy \& Araújo, 2009) sobre el tema, les fue solicitado a los estudiantes que se integraran en equipos de 3 y 4 miembros. Luego se les instruyó para, que, basados el desempeño académico y características personales, efectuaran la asignación de los roles de «líder de equipo», «secretario»y «gestor del tiempo». 
Análisis del problema a resolver. Para identificar las experiencias, conocimientos y habilidades de los estudiantes en la solución de un problema real y la contribución en su formación profesional, se diseñó un caso con la temática de Mercadotecnia Verde, la cual consideró los resultados de un estudio previo en el que se analiza el comportamiento de los consumidores verdes.

«Caso. La Mercadotecnia Verde (MV) en segmentos de Jóvenes Consumidores.

"Un estudio sobre la estructura de información sobre Mercadotecnia Verde concluyó que la información que determina las decisiones de compra de los consumidores está definida por segmentos clasificados por su intensidad al consumo verde, prácticas ecológicas, enfoque al medio ambiente, sensibilidad a la ecología y comportamiento del consumo verde fundamentados en estos comportamientos. Asimismo, el estudio plantea que la estructura de información sobre Mercadotecnia Verde (MV) en segmentos de Jóvenes Consumidores se encuentra en una etapa de transición. Aunque se encontró que un 41\% se preocupa por el medio ambiente, sólo $3 \%$ realiza cambios profundos en sus hábitos y menos del 50\% tiene disposición a recibir información ecológica o a pagar sobreprecio de productos. En suma, menos del 5\% es sensible a los temas ecológicos por lo que es difícil esperar un elevado consumo de Productos Verdes. Sin embargo, los segmentos también representan una oportunidad para incorporar procesos de aprendizaje relativos a las estructuras de información sobre Mercadotecnia Verde (MV). Con ello, se educaría a los consumidores sobre productos orgánicos, con sello verde, desechables reciclados, fabricados con productos naturales, conciencia verde, etc., ya que el bajo nivel informativo acerca del tema resulta poco rentable para la gestión comercial de las organizaciones”» (Lozano, 2018, p. 219).

Conclusión de la aplicación de la estrategia didáctica Aprendizaje Basado en Problemas. Para finalizar la práctica, se les requirió identificar el problema, la información requerida para su solución y la descripción detallada del mismo.

Resultados de la aplicación de la estrategia didáctica Aprendizaje Basado en Problemas. La aplicación de la estrategia didáctica tuvo una duración de 40 minutos aproximadamente. $\mathrm{Al}$ comienzo, los alumnos mostraron un comportamiento sumamente dinámico. Al paso del tiempo, su comportamiento se fue modificando ya que en algunos miembros hubo exceso de confianza en el tiempo y otros buscaban hacer prevalecer su opinión. Conforme el tiempo fue avanzado y al observar que no formulaban el problema, los integrantes de algunos equipos mostraron actitudes de ansiedad y disgusto. Sin embargo, retomaron las instrucciones y plantearon el problema a resolver. En esta etapa fue muy interesante analizar a los equipos cuyo planteamiento fue erróneo ya que no se correspondía con la realidad. $\mathrm{Al}$ finalizar del ejercicio los estudiantes expusieron que se les dificultó aceptar las aportaciones de sus compañeros y ello les restó tiempo para analizar el caso. Asimismo, se sintieron presionados por el tiempo y porque percibieron que al inicio del ejercicio su rol (líder, secretario, gestor del tiempo) no fue respetado. Aquí es pertinente reflexionar en que esta técnica demanda una interacción constante por parte de los integrantes del equipo, así como la reflexión, el debate, la exploración y la experimentación (Olmedo et al., 2016).

Seguimiento a la aplicación de la estrategia didáctica Aprendizaje Basado en Problemas. Para identificar la percepción y experiencias de los estudiantes en esta estrategia, se aplicó un cuestionario tipo escala Likert de 7 puntos en el cual se profundiza sobre su experiencia.

\section{Resultados}

\subsection{Pruebas no paramétricas}

Para identificar posibles relaciones entre las variables Aprendizaje Basado en Problemas y Adquisición de conocimientos y habilidades se aplicó una prueba de asociación (Ji-Cuadrada de independencia) a los 43 ítems que integran el Cuestionario. Aquí se consideró la Adquisición de conocimientos para la definición de un problema (P29) como pregunta principal para los ítems de cada variable (Aprendizaje Basado en Problemas y Adquisición de conocimientos y habilidades). Para esta prueba, las frecuencias esperadas ( $f e$ ), se comparan con las observadas ( $f o$ ) en la tabulación cruzada para calcular el estadístico (Levin \& Rubin, 2004; Malhotra, 2004; Moore, 2005; Hernández, Fernández, \& Baptista, 2010), por medio de la siguiente fórmula: 


$$
X^{2}=\sum \frac{(o-e) 2}{e}
$$

La frecuencia de cada celda se calcula con la siguiente fórmula:

$n_{r}=$ Número total en la fila

$n_{c}=$ Número total en la columna

$n=$ Tamaño de la muestra

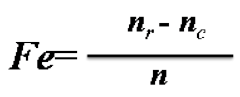

Los resultados de las pruebas reportan asociación a $X^{2}$ en 29 de 36 variables (figura 1).

Figura 1.

Prueba de Asociación a $x^{2}$

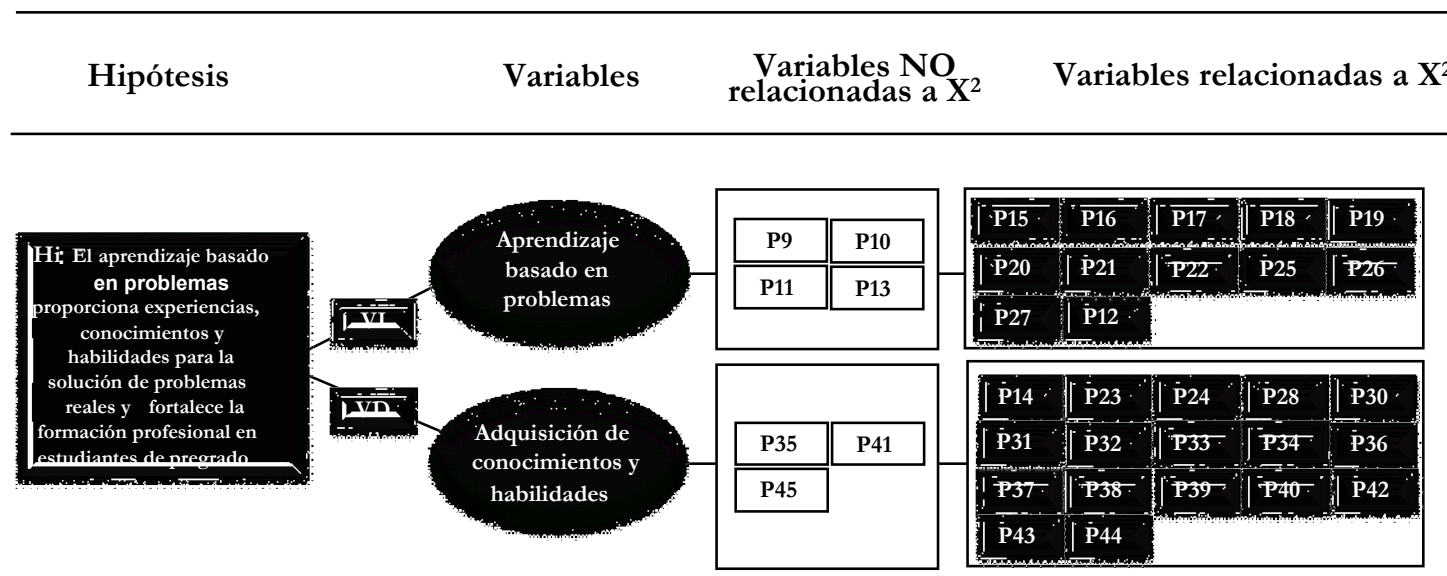

Fuente: elaboración propia.

\subsection{Demografía de la muestra}

Las características demográficas de la muestra corresponden a estudiantes que cursan el 4. ${ }^{\circ}$ semestre de la Licenciatura en Mercadotecnia. De esta muestra, el 61 \% son mujeres y $39 \%$ hombres, con rangos de edad entre los 21 y 25 años y un estado civil de soltero, principalmente. De lo anterior, un $47 \%$ trabaja a tiempo parcial en puestos de administrador, asesor de ventas o asistente.

\subsection{Aprendizaje basado en problemas}

Esta variable se integró por 16 ítems que abordan el análisis, la reflexión y la toma de decisiones respecto a un problema. Los resultados reportan un valor $p<.005$ rechaza $H o$ y explican asociación a $X^{2}$ en 12 de 16 variables. Esto indica que la adquisición de conocimientos para definir un problema, está relacionada con las decisiones para solucionarlo basadas en la definición de metas y expectativas, de manera objetiva, ética, reflexiva, crítica o creativa, partiendo del nivel de conocimientos de los integrantes del equipo, lo cual permite la asignación de tareas (figura 2). 
Figura 2.

Figura 2. Prueba de Asociación a $X^{2}$ de la variable Independiente: Aprendizaje basado en problemas.

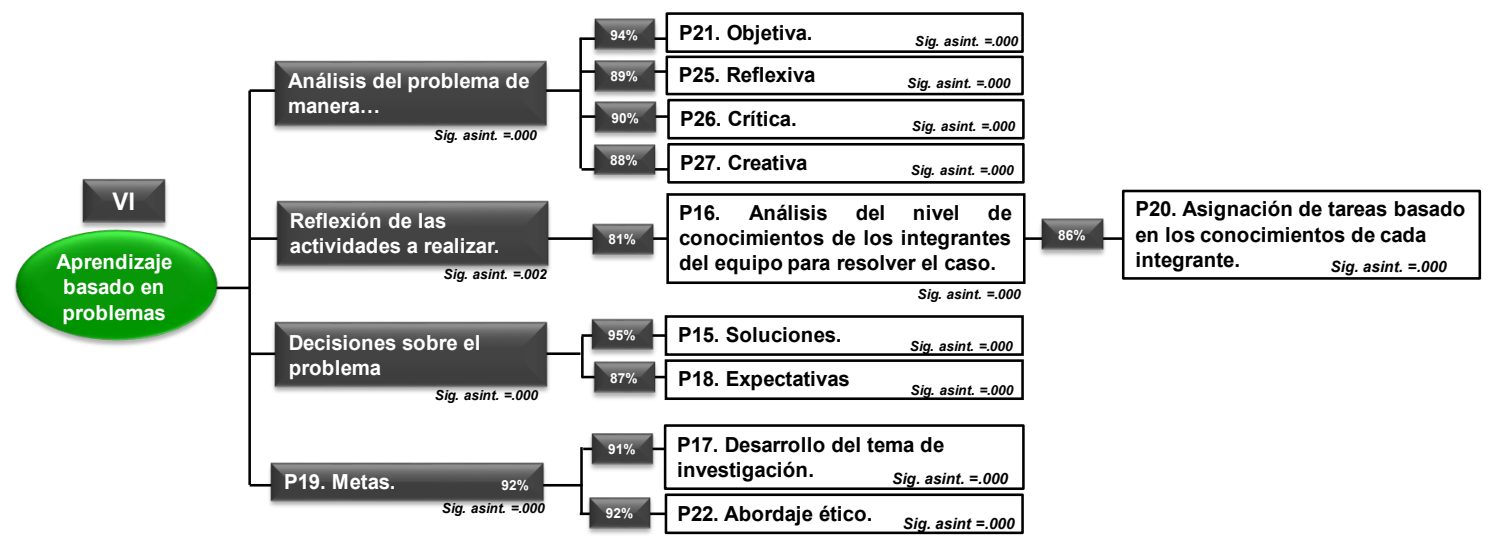

Fuente. Elaboración propia.

El constructivismo considera al alumno como responsable de su propio aprendizaje y del desarrollo y optimización de sus competencias. A su vez, al profesor lo enfoca como facilitador de la estrategia didáctica Aprendizaje Basado en Problemas en la que se combina la adquisición de conocimientos con el desarrollo de habilidades y actitudes. Ello permite que la reflexión se convierta en un hábito, otorgándole criterios para afrontar todas las situaciones que se le presenten en su vida personal y profesional (Gil, 2018:74, 75). En este punto, los estudiantes perciben que resolvieron el problema con objetividad porque en la asignación de tareas analizaron previamente el nivel de conocimientos de cada integrante, lo cual facilitó el desarrollo de actividades y la toma de decisiones respecto a qué y cómo resolver el problema, generar expectativas y metas (figura 3).

Figura 3.

Figura 3. Percepción de los estudiantes sobre sus actitudines en la solución del caso (Aprendizaje Basado en problenas).

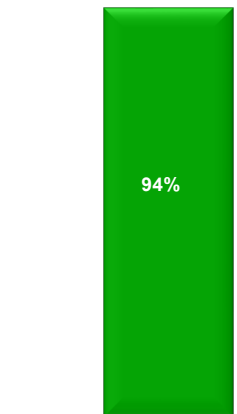

P21. Objetiva.

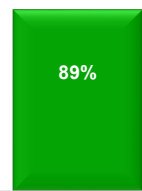

P25. Reflexiva

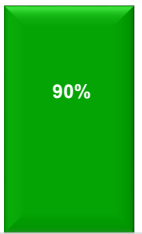

P26. Crítica.

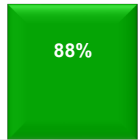

P27. Creativa 


\subsection{Adquisición de conocimientos y habilidades}

Esta dimensión se integró por 20 variables. Para este caso, los resultados de las pruebas indican relación entre las variables, el valor $p<.005$ rechaza Ho y asociación a $X^{2}$ en 17 de 20 variables, lo cual explica que los estudiantes adquieren conocimientos que les permiten estar en posibilidades de evaluar y solucionar problemas cotidianos, familiares o académicos. Esto favorece su formación profesional para el desarrollo de estrategias de estudio, comprensión de otras materias o argumentación de tareas escolares (figura 4).

Figura 4.

Figura 4. Prueba de Asociación a $X^{2}$ de la variable dependiente: Variable. Adquisición de conocimientos y habilidades.

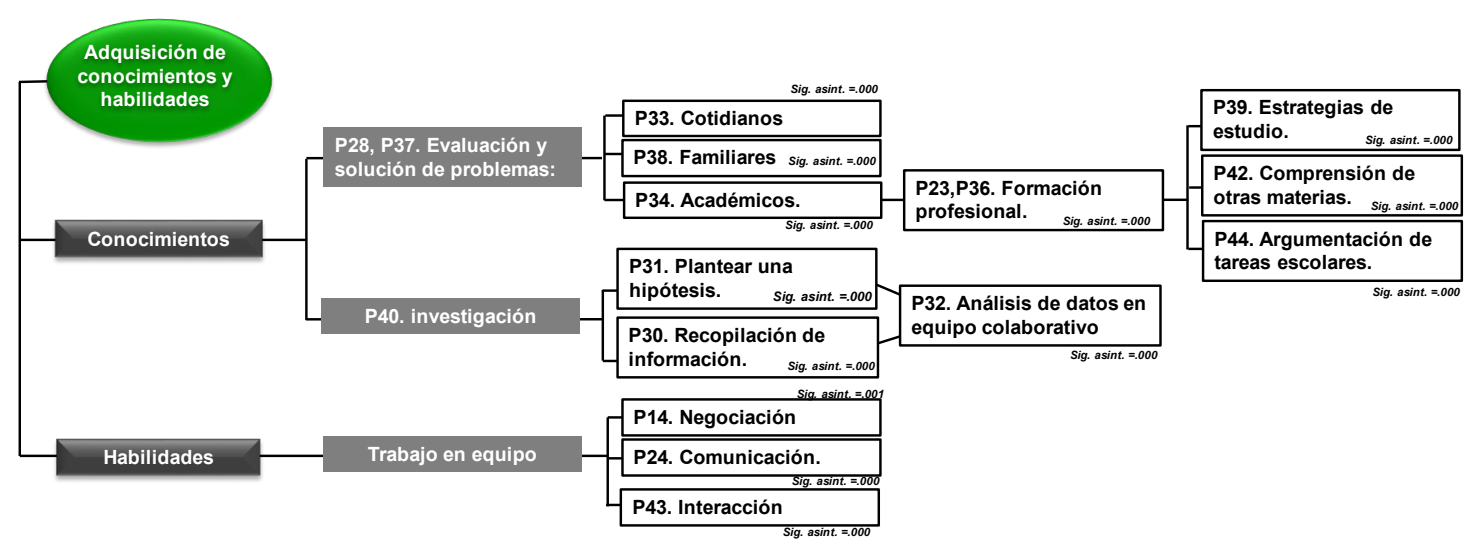

Fuente. Elaboración propia.

Ahora, de acuerdo a la escala Likert, las distribuciones de frecuencias muestran que las respuestas de los estudiantes se ubican en las puntuaciones más altas. Esto es muy importante para la evaluación y solución de problemas porque en las respuestas se refleja que un $96 \%$ percibe que adquirió conocimientos para la evaluación y solución de problemas que se les presenten. Estos conocimientos les permitirán desarrollar las actividades de aprendizaje en equipo de manera estratégica, evitando la confrontación con sus compañeros. Así también, estos conocimientos y habilidades les servirán al momento en que se integren a la vida laboral porque el perfil de su carrera les demanda trabajo coordinado (figura 5).

Figura 5.

Figura 5. Conocimientos adquiridos por los estudiantes para la evaluación y solución de problemas

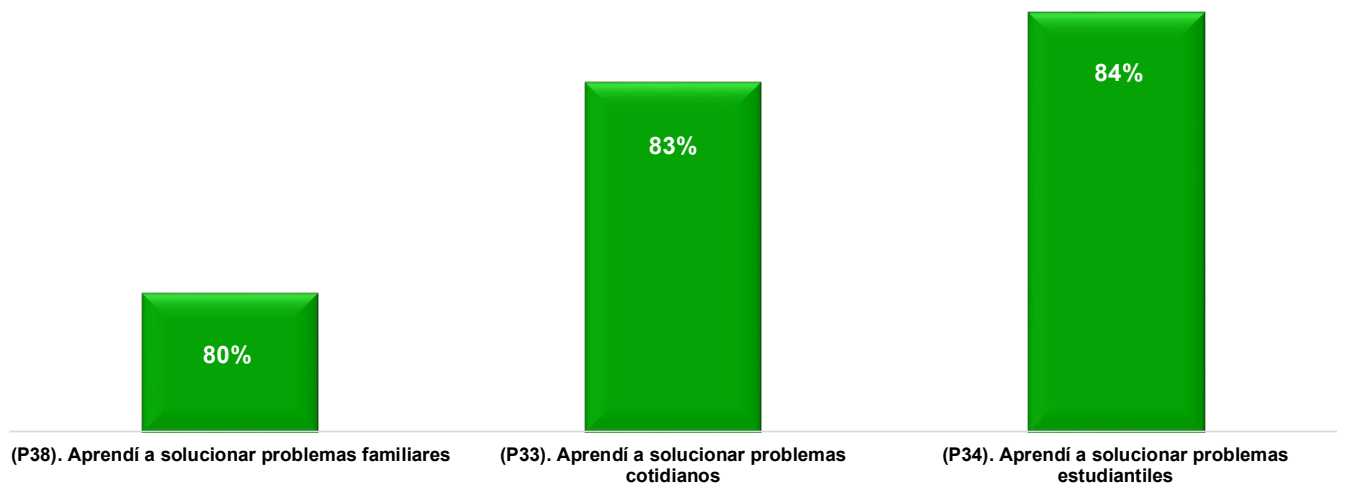


En lo relativo a las actividades de investigación, el $88 \%$ de los estudiantes considera que adquirió conocimientos para plantear una hipótesis, recopilar información y analizar datos en equipo. Por ello, un $92 \%$ tiene la certeza de que el ejercicio les ayudó a conocer y practicar habilidades de negociación con los integrantes del equipo, comunicarse e interactuar entre pares (figura 6). Cabe destacar que estos conocimientos apoyan el desarrollo de investigación para el $5 .^{\circ}$ semestre porque se les facilitará la formulación del problema y el diseño metodológico de la investigación. Lo anterior es muy importante porque en muchos casos la ausencia de comunicación les impide trabajar en equipo y a la mitad del semestre terminan realizando la investigación de manera individual o reprobando el curso.

Figura 6.

\section{Figura 6. Conocimientos adquiridos por los estudiantes para la evaluación y solución de problemas}

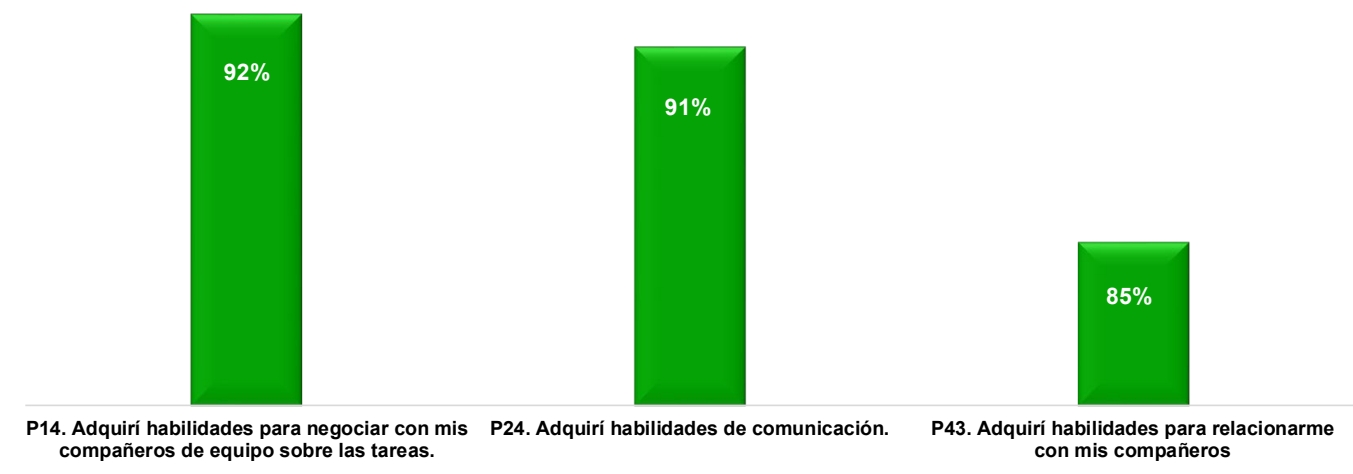

\subsection{Contrastación de hipótesis}

La Hipótesis de investigación se planteó como: «Hi: El aprendizaje basado en problemas proporciona experiencias, conocimientos y habilidades para la solución de problemas reales y fortalece la formación profesional en estudiantes de pregrado». Los resultados de las pruebas explican que el Aprendizaje basado en problemas proporciona:

- Experiencias que contribuyen a su formación profesional, sobre todo en la comprensión de otras materias, argumentación de tareas escolares y desarrollo de estrategias de estudio.

- Habilidades de negociación, comunicación, interacción, análisis en equipo, evaluar problemas, cotidianos, familiares y académicos.

- Conocimientos para realizar investigación (plantear hipótesis, recopilar información). 
Figura 7.

Figura 7. Contribución del Aprendizaje basado en problemas en la formación profesional de estudiantes de pregrado.

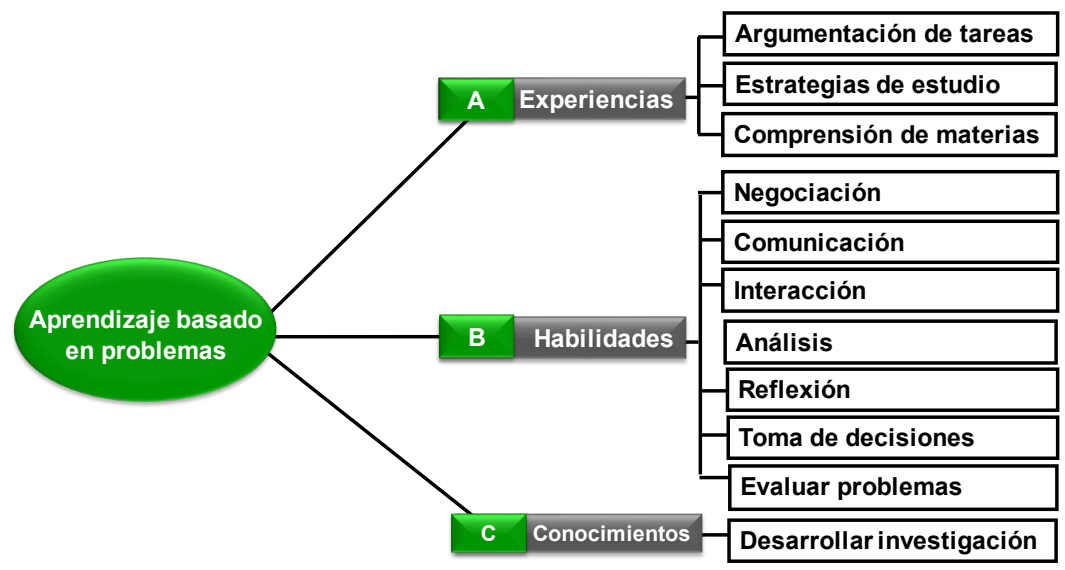

Fuente. Elaboración propia.

Estos resultados son consistentes con el estudio de (Gil, 2018:81), en el cual identificó que los estudiantes adquieren competencias técnicas a través del Aprendizaje basado en problemas en los contenidos de la asignatura, principalmente en el desarrollo de capacidades creativas, de análisis, de síntesis, evaluación y adquisición de conocimientos basados en problemas reales. Además de competencias metodológicas para solución de problemas, motivación para el aprendizaje, etc., y competencias participativas en la comunicación e interacción.

\section{Discusión y conclusiones}

Dado que el Aprendizaje basado en Problemas es un método de instrucción basado en la indagación, lo cual facilita el aprendizaje de los estudiantes a través de la colaboración (Galindo, et. al., 2011:328, 330; Lee, et. al., 2017:1629), consideramos que contribuye en la construcción de conocimientos y en el desarrollo de competencias de los estudiantes porque se requiere que identifiquen el problema, determinen lo que conocen y la información que necesitan para aportar soluciones por medio del pensamiento crítico y la creatividad.

Por consiguiente, la experiencia y conocimientos del profesor como facilitador de su aprendizaje es fundamental para un aprendizaje eficaz (Pazan y Flores, 2019:83). Por tanto, un vacío de conocimientos no respaldaría su aprendizaje (Sepúlveda, et. al., 2019:2). Por ello, la capacitación orientada hacia los profesores para que desarrollen sus habilidades e infraestructura para proporcionar asesoría en la implementación de esta estrategia es fundamental (Costas y Nicolaou, 2018:4).

Por consiguiente, se concluye que el Aprendizaje basado en Problemas contribuye en la formación profesional de los estudiantes de pregrado porque apoya sus procesos de aprendizaje para el análisis y reflexión de problemas. Sin embargo, como técnica es poco empleada por los profesores en el aula. Por ello, se recomienda efectuar un diagnóstico para conocer las estrategias didácticas implementadas por los profesores y desarrollar procesos de entrenamiento que apoyen su práctica docente.

\section{Anexo A}

Tabla A1.

Fundamentación teórica del articulo «El Aprendizaje basado en problemas en estudiantes de pregrado» 


\begin{tabular}{|c|c|c|c|c|c|}
\hline & Introducción & $\begin{array}{c}\text { Perspectiva } \\
\text { teórica del } \\
\text { Aprendizaje } \\
\text { Basado en } \\
\text { Problemas }\end{array}$ & Método & $\begin{array}{l}\text { Resulta- } \\
\text { dos }\end{array}$ & $\begin{array}{c}\text { Discusión y } \\
\text { Conclusio- } \\
\text { nes }\end{array}$ \\
\hline $\mathrm{ABP}, \mathrm{s} / \mathrm{f}$ & & $\mathrm{X}$ & & & \\
\hline Costas y Nicolaou, 2018 & $\mathrm{X}$ & & & & \\
\hline $\begin{array}{l}\text { Barbosa, Kruta, Rodríguez } \\
\text { y Felipe, } 2018\end{array}$ & & $\mathrm{X}$ & & & \\
\hline Carrasco, 2004 & & $\mathrm{X}$ & & & \\
\hline $\begin{array}{l}\text { Carrió, Agell, Rodríguez, } \\
\text { Larramona, Pérez y Baños, } \\
2018\end{array}$ & & $\mathrm{X}$ & & & \\
\hline Costas y Nicolaou, 2018 & & $\mathrm{X}$ & & & $\mathrm{X}$ \\
\hline Campoy y Araújo, 2009 & & & $\mathrm{X}$ & & \\
\hline Ding, 2016 & & $\mathrm{X}$ & & & \\
\hline Dmitrenko, 2016 & & $\mathrm{X}$ & & & \\
\hline $\begin{array}{l}\text { Edwards, Kupczynski y } \\
\text { Groff, } 2019\end{array}$ & & $\mathrm{X}$ & & & \\
\hline Etherington, 2011 & $\mathrm{X}$ & & & & \\
\hline $\begin{array}{l}\text { Fernández, Fernández y } \\
\text { Polo, } 2017\end{array}$ & $\mathrm{X}$ & $\mathrm{X}$ & & & \\
\hline Feo, 2010:222 & & $\mathrm{X}$ & & & \\
\hline $\begin{array}{l}\text { Gómez, } 2005 \text { cit. en Rodrí- } \\
\text { guez, Ordoñez y Meneses, } \\
2018\end{array}$ & $\mathrm{X}$ & & & & \\
\hline $\begin{array}{l}\text { Galindo, Arango, Díaz, Vi- } \\
\text { llegas, Aguirre, Kam- } \\
\text { bourova y Jaramillo, } 2011\end{array}$ & $\mathrm{X}$ & & & & $\mathrm{X}$ \\
\hline Gil, 2018 pp. 74,75 & & & & $\mathrm{X}$ & \\
\hline $\begin{array}{l}\text { Giraldo, Giraldo y Valde- } \\
\text { rrama, } 2018\end{array}$ & $\mathrm{X}$ & & & & \\
\hline Gorbaneff, 2010 & $\mathrm{X}$ & & & & \\
\hline Gros, 1990 & $\mathrm{X}$ & & & & \\
\hline Gregori y Menéndez, 2015 & & $\mathrm{X}$ & & & \\
\hline $\begin{array}{l}\text { González, Carbonero, Lara } \\
\text { y Martín, } 2014\end{array}$ & $\mathrm{X}$ & & & & \\
\hline Gil 2018 & $\mathrm{X}$ & & & & \\
\hline $\begin{array}{l}\text { Geitz, Brinke y Kirschner, } \\
2016\end{array}$ & & $\mathrm{X}$ & & & \\
\hline $\begin{array}{l}\text { Hemker, Prescher y Nar- } \\
\text { ciss, } 2017\end{array}$ & $\mathrm{X}$ & & & & \\
\hline Hemker, et. al., 2017 & & $\mathrm{X}$ & & & \\
\hline $\begin{array}{l}\text { Hernández, Fernández y } \\
\text { Baptista, } 2010\end{array}$ & & & & $\mathrm{X}$ & \\
\hline $\begin{array}{l}\text { Lee, Lajoie, Poitras, } \\
\text { Nkangu, \&Tenzin, } 2017\end{array}$ & & $\mathrm{X}$ & & & $\mathrm{X}$ \\
\hline Levin y Rubin, 2004 & & & & $\mathrm{X}$ & \\
\hline $\begin{array}{l}\text { Lima, Carvalho, Sousa, } \\
\text { Arezes y Mesquita, } 2017\end{array}$ & & $\mathrm{X}$ & & & \\
\hline Malhotra; 2004 & & & & $\mathrm{X}$ & \\
\hline Makin, 2015 & & $\mathrm{X}$ & & & \\
\hline Moore, 2005 & & & & $\mathrm{X}$ & \\
\hline Ortega y Carrascal, 2017 & $\mathrm{X}$ & & & & \\
\hline $\begin{array}{l}\text { Olmedo, Alvarado, Del- } \\
\text { gado, Montero, Cadenas, } \\
\text { Mora y Hernández, } 2016\end{array}$ & & $\mathrm{X}$ & $\mathrm{X}$ & & \\
\hline Pazan y Flores, 2019 & & & & & $\mathrm{X}$ \\
\hline
\end{tabular}




\begin{tabular}{|c|c|c|c|c|c|}
\hline & Introducción & $\begin{array}{c}\text { Perspectiva } \\
\text { teórica del } \\
\text { Aprendizaje } \\
\text { Basado en } \\
\text { Problemas }\end{array}$ & Método & $\begin{array}{l}\text { Resulta- } \\
\text { dos }\end{array}$ & $\begin{array}{c}\text { Discusión y } \\
\text { Conclusio- } \\
\text { nes }\end{array}$ \\
\hline $\begin{array}{l}\text { Rodríguez, Pirul, Robles, } \\
\text { Pérez, Vásquez, Galaz, } \\
\text { Cuellar, Díaz y Arriaza, } \\
2018\end{array}$ & & $\mathrm{X}$ & & & \\
\hline Sainz y Fernández, 2012 & & $\mathrm{X}$ & & & \\
\hline $\begin{array}{l}\text { Sepúlveda, Cabezas, García } \\
\text { y Fonseca, } 2019\end{array}$ & & $\mathrm{X}$ & & & $\mathrm{X}$ \\
\hline $\begin{array}{l}\text { Teruel, Domínguez y Fer- } \\
\text { nández, } 2015\end{array}$ & & $\mathrm{X}$ & & & \\
\hline Travieso y Ortiz 2018 & & $\mathrm{X}$ & & & \\
\hline $\begin{array}{l}\text { Walker, Bridges y Chan, } \\
1996\end{array}$ & $\mathrm{X}$ & & & & \\
\hline
\end{tabular}

Fuente: elaboración propia.

Referencias

$\operatorname{ABP}(s / f)$. Las estrategias y técnicas didácticas en el rediseño. Dirección de Investigación y Desarrollo Educativo. Vicerrectoría Académica, Instituto Tecnológico y de Estudios Superiores de Monterrey. Disponible en http://sitios.itesm.mx/va/dide/documentos/inf-doc/abp.pdf.

Barbosa, D. S., Kruta, D. A. A. C., Rodríguez, G. D., \& Felipe, V. F. L. (2018). Problem-based learning: A proposal for structuring PBL and its implications for learning among students in an undergraduate management degree program. Revista de Gestão, 25(2), pp. 160-177. doi: 10.1108/REGE03-2018-030.

Calvo, V. M. (2005). Formador Ocupacional. Formador de formadores. Formación Profesional Ocupacional (FPO). España. Editorial Mad, SL.

Carrasco, J. B. (2004). Una didáctica para hoy: cómo enseñar mejor. España. Ediciones Rialp, SA.

Campoy, A. T. J. \& Araújo, G. E. (2009). Manual básico para la realización de tesinas, tesis y trabajos de investigación. Capítulo 10. Técnicas instrumentos cualitativos de recogida de datos. Editorial EOS. Disponible en http://www2.unifap.br/gtea/wp-content/uploads/2011/10/T_cnicas-einstrumentos-cualitativos-de-recogida-de-datos1.pdf.

Carrió, M., Agell, L., Rodríguez, G., Larramona, P., Pérez, J., \& Baños, J. E. (2018). Percepciones de estudiantes y docentes sobre la implementación del aprendizaje basado en problemas como método docente. FEM: Revista de la Fundación Educación Médica, 21(3). doi: 10.33588/fem.213.947.

Ding, X. W. (2016). The Effect of WeChat-assisted Problem-based Learning on the Critical Thinking Disposition of EFL Learners. International Journal of emerging technologies in learning, 11(12). doi: 10.3991/ijet.v11i12.5927.

Dmitrenko, N. (2016). The implementation of problem-based learning in Ukrainian higher educational institutions. Advanced Education, 5. doi: 10.20535/2410-8286.61834.

Edwards, D. J., Kupczynski, L., \& Groff, S. L. (2019). Learning Styles in Problem-based Learning Environments Impacts on Student Achievement and Professional Preparation in University Level Physical Therapy Courses. International Journal of Higher Education, 8(3). doi: 10.5430/ijhe.v8n3p206.

Etherington, M. B. (2011). Investigative Primary Science: A Problem-based Learning Approach. Australian Journal of Teacher Education, 36(9). doi: 10.14221/ajte.2011v36n9.2.

Fernández, J. C., López, J. M. D., Fernández, M. \& Polo, T. (2014). Aplicación del aprendizaje basado en problemas para la formación de alumnado de educación en la atención a la discapacidad. Revista Profesorado. Revista de currículum y formación de profesorado, 18(2).

Fernández, J. C., Fernández, C. M. \& Polo, S. M. T. (2017). Aplicación de la autoevaluación en una experiencia de Aprendizaje Basado en Problemas con alumnado de educación en asignaturas relacionadas con la discapacidad, Iatreia, 32, pp. 73-93. doi: 10.15581/004.32.73-93.

Feo, R. (2010). Orientaciones básicas para el diseño de estrategias didácticas. Revista tendencias pedagógicas, 16. 
Galindo, C. L. A., Arango, R. M. E., Díaz, H. D. P., Villegas, M., E. M., Aguirre, M. C. E., Kambourova, M. \& Jaramillo, M. P. A. (2011). ¿Cómo el aprendizaje basado en problemas (ABP) transforma los sentidos educativos del programa de Medicina de la Universidad de Antioquia?, Iatreia, 24(3), pp. 325-334.

Geitz, G. Brinke, D. J. \& Kirschner, P. A. (2016). Are marketing students in control in problembased learning? Revista Cogent Education. doi: 10.1080/2331186X.2016.1222983.

Gil, G. R. (2018). El uso del aprendizaje basado en problemas en la enseñanza universitaria. Análisis de las competencias adquiridas y su impacto. Revista mexicana de investigación educativa, 23(76), pp.7393.

Giraldo, P. E. L., Giraldo, G. J. A. \& Valderrama, O. J. A. (2018). Modelo de Simulación de un Sistema Logístico de Distribución como Plataforma Virtual para el Aprendizaje Basado en Problemas. Revista Información Tecnológica, 29(6), pp. 185-198. doi: 10.4067/S071807642018000600185.

González, H. C., Carbonero, M. M. Á., Lara, O. F. \& Martín, V. P. (2014). Aprendizaje Basado en Problemas y satisfacción de los estudiantes de Enfermería. Revista enfermería Global, 35. doi: 10.6018/eglobal.13.3.175301.

Gorbaneff, Y. (2010). Qué se puede aprender de la literatura sobre el aprendizaje basado en problemas. Revista de la Facultad de Ciencias Económicas, XVIII(1), pp. 61-74. doi: 10.18359/rfce.2001.

Gregori, G. E. \& Menéndez, V. J. L. (2015). La percepción de los estudiantes de Bellas Artes sobre lo aprendido en un entorno de aprendizaje basado en problemas. Revista Mexicana de Investigación Educativa, 20(65), pp. 481-506.

Gros, S. B. (1990). La enseñanza de estrategias de resolución de problemas mal estructurados. Investigaciones y Experiencias. Universidad de Barcelona. Revista de Educación. Disponible en https://www.mecd.gob.es/dctm/revista-deeducacion/articulosre293/re2932000479.pdf?documentId=0901e $72 \mathrm{~b} 81377331$.

Hemker, L., Prescher, C. \& Narciss, S. (2017). Design and Evaluation of a Problem-Based Learning Environment for Teacher Training. Interdisciplinary Journal of Problem-based Learning, 11(2). doi: 10.7771/1541-5015.1676.

Hernández, S. R., Fernández-Collado, C. \& Baptista, L. P. (2010). Metodología de la investigación. Mc Graw-Hill Interamericana Editores, S. A. de C. V. México.

Lee, L., Lajoie, S. P., Poitras, E. G., Nkangu, M. \& Tenzin Doleck, T. (2017). Co-regulation and knowledge construction in an online synchronous problem-based learning setting. Educ Inf Technol (2017), 22. doi: 10.1007/s10639-016-9509-6.

Levin, R. I. \& Rubin, D. S. (2004). Estadística para administración y economía. México. Editorial Pearson Educación.

Lima, R. M., Carvalho, J. C., Sousa, R. M., Arezes, P., \& Mesquita, D. (2017). Development of competences while solving real industrial interdisciplinary problems: a successful cooperation with industry. Production, 27(No. spe). doi: 10.1590/0103-6513.230016.

Lozano, R. M. C. (2018). Gestión del conocimiento para el diseño de estructuras de información sobre Mercadotecnia Verde (MV) en segmentos de Jóvenes Consumidores. Revista El Periplo sustentable, 34, pp. 214-235.

Makin, D. A. (2015). A Descriptive Analysis of a Problem-Based Learning Police Academy. The Interdisciplinary Journal of Problem-based Learning, 10(1). doi: 10.7771/1541-5015.1544.

Malhotra, N. K., (2004). Investigación de mercados. México. Pearson Educación.

Moore, D. S. (2005). Estadística aplicada básica. España. Editorial Antoni Bosch.

Olmedo, B. B. A., Alvarado, B. H. M., Delgado, E. I., Montero, C. S. A., Cadenas, F. J. L., Mora, B. A. B. \& Hernández, T. E. (2016). Desempeño estudiantil con el aprendizaje basado en problemas: habilidades y dificultades. Revista Cubana de Medicina General Integral, 32(2).

Ortega, A. S. P. \& Carrascal, T. S. N. (2017). Aprendizaje basado en problemas como estrategia para el desarrollo de competencias económicas y financieras desde el álgebra. Revista Diálogo. Canoas, 36, pp. 191-201. doi: 10.18316/dialogo.v0i36.4305.

Pazan, E. G., \& Flores, J. R. (2019). El Aprendizaje Basado en Problemas y el Uso del Paquete Estadístico $R$ en la Interpretación de las Gráficas de Control. Espirales Revista multidisciplinaria de investigación cientifica, 3(26). doi: 10.31876/re.v3i26.462. 
Rodríguez, M. B. J., Ordoñez, D. M. M. \& Meneses, S. L. C. (2018). Strengthening of Reasoning Levels in higher education students through the use of Learning Strategies (Problem-Based Learning and Collaborative Learning) Using ICT's. Electronic Journal of Research in Educational Psychology, 16(45), pp. 477-502. 2018. doi: 10.25115/ejrep.v16i45.2102.

Rodríguez, H., Pirul, J., Robles, J., Pérez, L., Vásquez, E., Galaz, I., Cuellar, C., Díaz, H. \& Arriaza, C. (2018). Análisis de los estilos de aprendizaje en alumnos de Medicina de la Universidad de Chile. Revista Educación Médica, 19(1), pp. 2-8. doi: 10.1016/j.edumed.2016.11.004.

Sainz, S. C., \& Fernández, R. S. (2012). Pensamiento crítico y aprendizaje basado en problemas cotidianos. Revista de Docencia Universitaria, 10(3), pp. 325-346. doi: 10.4995/redu.2012.6026.

Sepúlveda, P., Cabezas, M., García, J. \& Fonseca, S. F. (2019). Aprendizaje basado en problemas: percepción del proceso enseñanza aprendizaje de las ciencias preclínicas por estudiantes de Kinesiología. Revista Educación Médica. Elsevier España. doi: 10.1016/j.edumed.2019.01.004.

Teruel, M. M., Domínguez, M. M. \& Fernández, F. E. (2015). Estrategia didáctica con el apoyo de las TICS para el desarrollo de habilidades profesionales en la licenciatura en contabilidad y finanzas en el modelo semipresencial. Revista de Pedagogía Universitaria, 20(1), pp. 71-81.

Travieso, V. D. \& Ortiz Cárdenas T. (2018). Aprendizaje basado en problemas y enseñanza por proyectos: alternativas diferentes para enseñar. Revista Cubana de Educación Superior, 37(1), pp. 124-133.

Walker, A., Bridges, E., \& Chan, B. (1996). Wisdom gained, wisdom given: instituting PBL in a Chinese culture. Journal of Educational Administration, 34(5), pp. 12-31. doi: 10.1108/09578239610148250. 